\title{
Facomatosis pigmentovascular cesioflammea asociada a epilepsia focal mioclónica: Primer caso reportado en el Perú
}

Phakomatosis pigmentovascularis cesioflammea associated with focal myoclonic seizures: First case report in Peru

\author{
Miguel A. Vences ${ }^{1, a}$, Elliot Barreto-Acevedo ${ }^{1, b}$, Fiorella Delgado-Acosta ${ }^{2, a}$
}

\section{RESUMEN}

La facomatosis pigmentovascular es un síndrome congénito muy poco frecuente, caracterizado por la presentación simultánea de una malformación vascular capilar y una lesión cutánea pigmentaria, con o sin compromiso extracutáneo. Se presenta el caso de una adolescente con epilepsia que cursa con crisis mioclónicas focales no controladas por un tratamiento farmacológico irregular, y que muestra además lesiones cutáneas compatibles con nevus flammeus y melanosis dérmica, ocular y palatina, presentes desde el nacimiento. Se trata del primer reporte en el país, de un síndrome neurocutáneo poco frecuente y de su asociación clínica con epilepsia, resaltándose además la importancia de una evaluación integral de esta entidad.

PALABRAS CLAVE: Epilepsia focal; facomatosis cesioflammea; facomatosis pigmentovascular; mioclonías.

\section{SUMMARY}

Phacomatosis pigmentovascularis is a rare congenital syndrome, characterized by the simultaneous presentation of a capillary vascular malformation and a cutaneous pigmentary lesion, without or with extracutaneous involvement. The case of an adolescent with epilepsy characterized by focal myoclonic seizures uncontrolled by an irregular pharmacological treatment, with skin lesions compatible with nevus flammeus and dermal, ocular and palatal melanosis since birth, is presented. This is the first report in the country of an infrequent neurocutaneous syndrome and its clinical association with epilepsy, highlighting besides the importance of a comprehensive evaluation of this entity.

KEYWORDS: Focal epilepsy; phacomatosis cesioflammea; phacomatosis pigmentovascularis; myoclonic seizures.

1 Departamento de Neurología, Servicio de Epilepsia y Enfermedades Cerebrovasculares. Hospital Nacional Edgardo Rebagliati Martins, Essalud. Lima. Perú.

2 Departamento de Dermatología, Hospital Nacional Edgardo Rebagliati Martins, Essalud. Lima, Perú.

a Médico residente; ${ }^{\mathrm{b}}$ Médico Neurólogo Asistente. 


\section{INTRODUCCIÓN}

La facomatosis pigmentovascular es un síndrome congénito raro, caracterizado por la presentación simultánea de una malformación vascular capilar con una lesión pigmentaria cutánea, con o sin compromiso extracutáneo (1). El tipo cesioflammea es el más frecuente y constituye la asociación de melanocitosis dérmica y nevus flammeus, también llamado mancha en vino de Oporto (2). En los sindromes neurocutáneos se suelen presentar crisis epilépticas generadas por alteración cortical asociada a compromiso vascular yuxtacortical o por asociación a displasias o microdisplasias corticales.

El objetivo del presente caso es reportar la primera descripción en el país de facomatosis pigmentovascular cesioflammea y su asociación clínica con epilepsia, realizando una caracterización clínica de esta entidad y resaltando la importancia de una evaluación integral en los pacientes con síndromes neurocutáneos.

\section{Presentación del caso}

Paciente de sexo femenino de 17 años de edad, nacida de parto eutócico y con desarrollo psicomotor adecuado, nacida de padres no consanguíneos y sin antecedentes familiares significativos. Presenta desde el nacimiento diferentes máculas cutáneas eritematosas y grises-azuladas distribuidas en cara, tronco y extremidades (figura 1 (a y b)). La paciente es referida a la Unidad de Epilepsia con el diagnóstico de epilepsia mioclónica juvenil farmacorresistente, caracterizada por crisis mioclónicas que presentaba desde los 12 años. En el último año la epilepsia no era controlada a pesar de tratamiento farmacológico habitual con Valproato $1000 \mathrm{mg}$ y Lamotrigina 100 $\mathrm{mg}$ diarios, el cual fue el régimen establecido desde el diagnóstico inicial.

Al ingreso se realizó una ampliación de la anamnesis; dichos eventos paroxismales en un inicio se caracterizaban por mioclonías localizadas en el

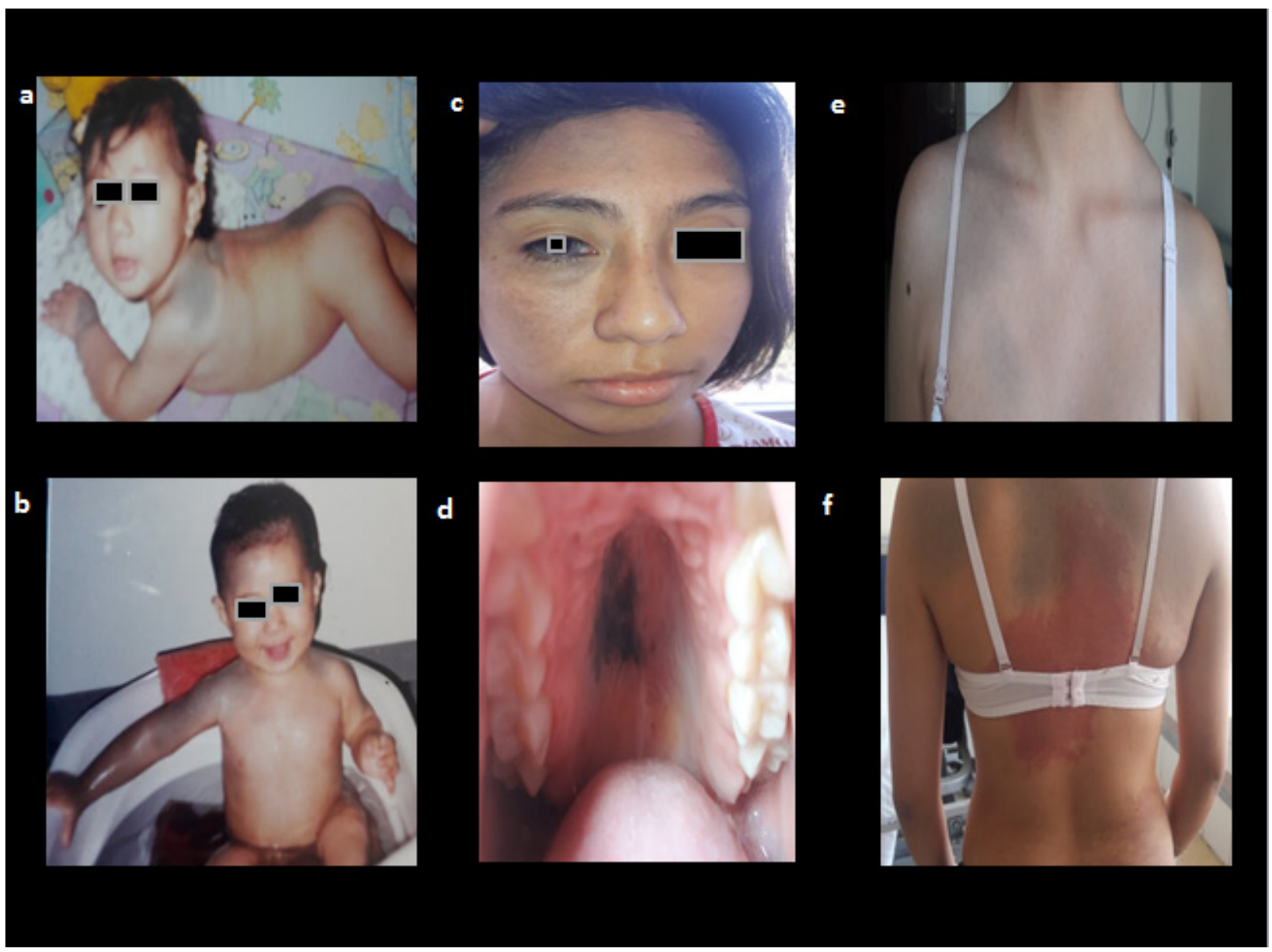

Figura 1. Se muestra en a y b) Melanosis dérmica en miembro superior derecha, región acromioclavicular, escapular y lumbosacra, asociada a nevus flammeus en región dorsal. c) Nevus de Ota. d) Melanosis de paladar. e) Nevus de Ito. f) Melanosis dérmica y nevus flammeus en tórax posterior. Nevus anemicus en región lumbar. 
hemicuerpo derecho sin progresión Jacksoniana $\mathrm{y}$ posteriormente se tornaron bilaterales en su mayoría, sin comprometer la conciencia, sin estar precedidas de síntomas prodrómicos, ni auras ni presentar déficit postictal. En el último año reportaron en 4 oportunidades crisis epilépticas tónico - clónicas de inicio desconocido y un aumento de la frecuencia de las crisis mioclónicas (dos a tres crisis diarias) que generaban caídas o que suelte objetos sujetados con las manos. Las crisis tenían presentación predominantemente diurna y no se disparaban por algún estímulo específico.

La exacerbación de las crisis ocurrió en un contexto de un cuadro de trastorno conductual y un síndrome ansioso depresivo caracterizado por angustia, anhedonia, agresividad, supresión de sueño y un episodio de fuga del domicilio. Por ello es internada en un centro de salud mental donde realizan despistaje de uso de drogas siendo positivo en examen de orina al uso de cannabinoides (tetrahidrocanabinol). Se realiza el diagnóstico de depresión mayor e inician medicación farmacológica con Sertralina y Risperidona, una vez mejorado su estado emocional y conducta es referida a la Unidad de Epilepsia y en nuestro servicio se documentó, además una muy pobre adherencia a los antiepilépticos desde hace 10 meses.

Se realizó un examen físico completo de la paciente, el examen neurológico fue normal y la evaluación

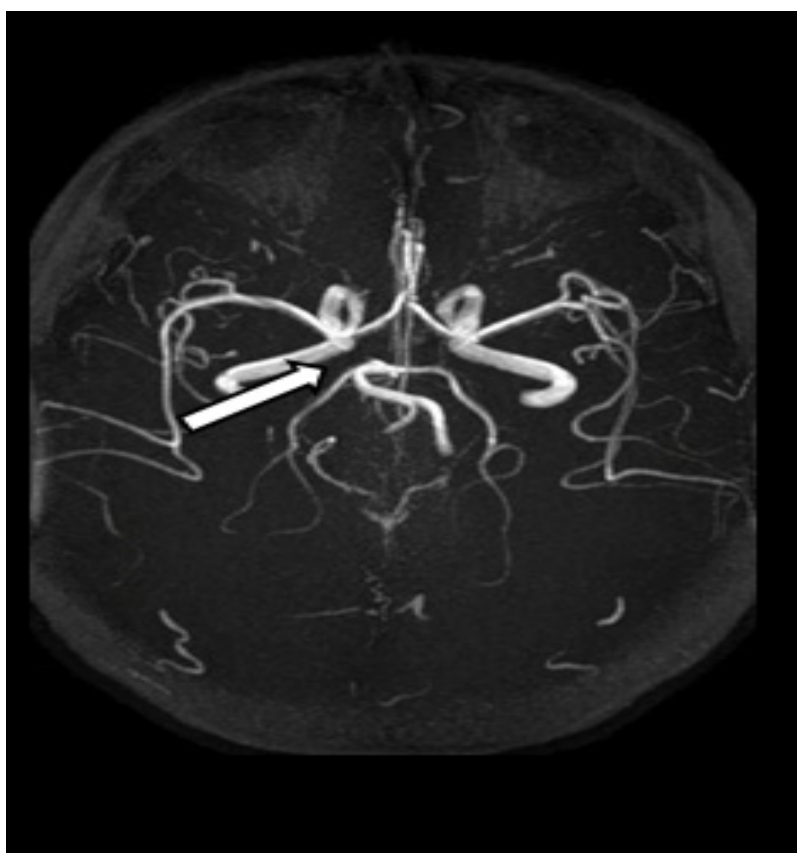

Figura 2. Angio Resonancia Cerebral. Hipoplasia de arteria comunicante posterior derecha. neuropsicológica no evidenció trastorno en las esferas cognitivas en general, la memoria verbal y pictórica mostraron un rendimiento promedio; además se identificó un síndrome ansioso-depresivo moderado. Al examen dermatológico realizado por el especialista, se evidenció melanosis en hemicara derecha y esclera ipsilateral compatible con Nevus de Ota (figura 1 (c)), melanosis en región de paladar duro [figura $1 \mathrm{~d}$ ] y Nevus de Ito en región acromioclavicular bilateral (figural (e)). Asimismo, se percibieron máculas eritematosas de bordes irregulares compatibles con nevus flammeus, distribuidas principalmente en región lumbo-sacra y nevus anemicus superpuestos en algunos sectores con manchas mongólicas aberrantes (figura 1 (f)), el resto del examen de aparatos y sistemas fue normal.

Con el objetivo de descartar compromiso extracutáneo agregado se realizó un examen oftalmológico que mostró agudeza visual, campimetría visual, fondo de ojo, diámetro corneal y presión intraocular normales en ambos ojos. Además, se realizó resonancia magnética de encéfalo con protocolo para epilepsia y angiorresonancia, destacándose hipoplasia de la arteria comunicante posterior derecha y se excluyó displasia cortical u otra anormalidad parenquimal (figura 2).

El video electroencefalograma de superficie de 42 horas con 30 minutos, en vigilia y sueño espontaneo evidenció actividad de fondo normal, escasa actividad epileptiforme interictal (paroxismos menor a dos segundos de ondas agudas y complejos punta ondas lentos 2 a $2,5 \mathrm{~Hz}$ ) con distribución frontal bilateral (mayor electronegatividad en Fp1 y Fp2) y fenómeno de sincronía bilateral secundaria en sueño y vigilia. Las maniobras de activación no generaron respuestas anormales. Se registraron dos crisis clínicas focales sin alteración de conciencia, mioclónicas (una con afectación de miembro superior izquierdo y otra con salva de mioclonías que comprometían al miembro superior derecho e inmediatamente se tornaron bilaterales en ambos miembros superiores). La actividad eléctrica ictal fue similar a la interictal; concluyendo en zona epileptógena frontal mesial sin una lateralización definitiva (figura 3).

Basado en la clínica, hallazgos y exámenes auxiliares, se concluyó en el diagnóstico de síndrome neurocutáneo: Facomatosis pigmentovascular cesioflammea asociada a epilepsia focal estructural y depresión asociada. Se optimizó la terapia antiepiléptica en monoterapia con Lamotrigina 150 


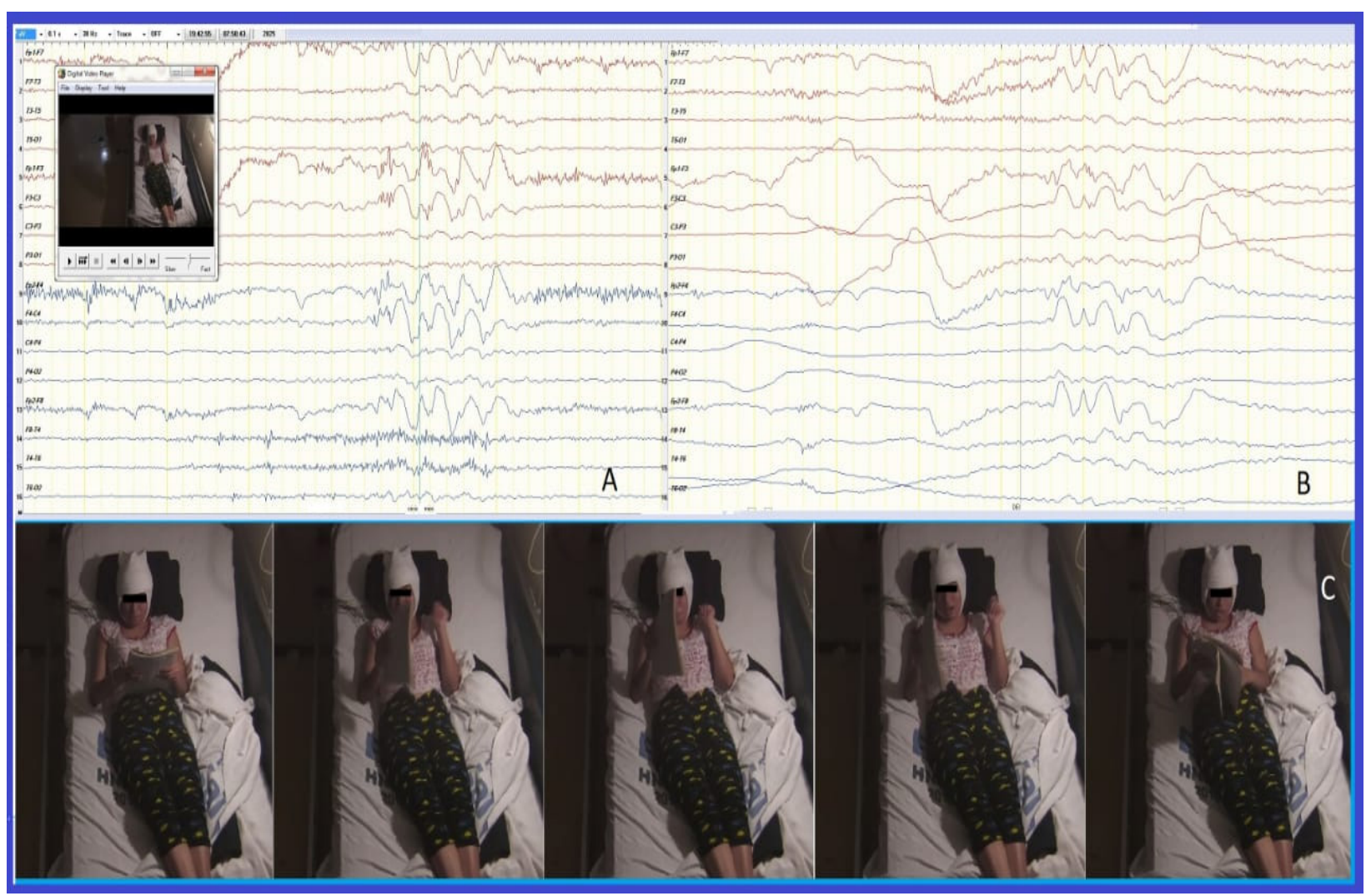

Figura 3. Video Electroencefalograma. a) Descarga ictal. b) Descarga interictal. c) Secuencia de crisis mioclónica con afectación de miembro superior izquierdo.

mg diarios dividida en dos dosis por ser un caso de pseudofarmacorresistencia. Se prefirió Lamotrigina frente al Levetiracetam y Topiramato debido al trastorno conductual y emocional previo; además se decidió la suspensión de Valproato debido a ser una mujer en edad fértil.

En el seguimiento ambulatorio por neurología y salud mental, la paciente se encuentra libre de crisis epilépticas luego de dieciocho meses del alta, con mejoría del trastorno emocional, buena adherencia y sin consumo de drogas.

\section{DISCUSIÓN}

La Facomatosis pigmentovascular es un síndrome congénito que se caracteriza por la presencia de un nevus vascular asociado a un nevus pigmentario, fue descrita inicialmente en 1947 por Ota y desde entonces se han reportado pocos casos $(1,2)$. Originalmente fue clasificada en cuatro tipos (I - IV) por Hasegawa y Yasuhara y en el 2003, Torrelo agregó el tipo V descrito como cutis marmorata telangiectática congénita asociado a manchas mongólicas aberrantes; además, se clasificó en "a" y "b" de acuerdo a si existía únicamente compromiso cutáneo o presentaba compromiso extracutáneo (3). Posteriormente, en el 2005 Happle propuso una nueva clasificación: Facomatosis cesioflammea, spilorosea, cesiomarmorata y formas inclasificables de facomatosis (4).

La frecuencia de esta entidad es desconocida, debido a que la mayoría de estudios provienen de reportes de caso, habiéndose reportado a la fecha poco más de 200 casos, la mayoría en Japón y la India; el tipo cesioflammea es el tipo más frecuente representando alrededor del $70 \%$ de todos los casos de facomatosis pigmentovascular (5). En el 2002, en un estudio realizado en México reportó una serie de 24 pacientes con una frecuencia relativa de 5,8 por cada 100000 pacientes pediátricos atendidos en su institución, siendo la segunda serie más grande luego de una serie de Argentina con 25 casos; la existencia de casos en esta parte del continente americano, podría estar relacionada al fenómeno migratorio de poblaciones asiáticas $(6,7)$.

Su patogenia aún no se encuentra bien establecida, se piensa que existiría un defecto en el desarrollo de las células del nevus melanocítico y células neurales 
vasomotoras derivadas de la cresta neural, el cual se explicaría por un fenómeno llamado "twin spots" (manchas gemelas) o didimosis, un mecanismo específico de mosaicismo somático (8), esto daría como resultado la presencia en una misma región del tegumento, de dos áreas de tejido mutante, distintas entre sí, y de tejido circundante normal (9). Además, se han identificado mutaciones asociadas en el gen GNA11 y GNAQ, genes que codifican subunidades $\mathrm{G} \alpha$ de proteínas $\mathrm{G}$ heterotriméricas (10).

Clasificamos a nuestro paciente como una Facomatosis cesioflammea de acuerdo a la clasificación de Happle o tipo IIb de acuerdo a la clasificación tradicional basado en los hallazgos clínicos: Nevus de Ota, melanosis oculi, Nevus de Ito, nevus flammeus y nevus anemicus, asociados como manifestación extracutánea a una epilepsia focal mioclónica y una hipoplasia de la arteria comunicante posterior derecha, ipsilateral al compromiso mayoritario de las lesiones dérmicas. Las lesiones dérmicas en algunos casos tienden a atenuarse en el transcurso de los años tal como lo sucedido con nuestra paciente con el nevus flammeus de la hemicara derecha, sin embargo, en nuestra paciente el resto de nevus flammeus y de manchas mongólicas aberrantes se han mantenido en el tiempo.

En nuestro caso se excluyeron en el diagnóstico diferencial otros síndromes cutáneos que están asociados a epilepsia tales como el síndrome de PHACE debido a la ausencia de hemangiomas segmentarios en piel y en otros órganos, además principalmente porque el tipo de lesión pigmentaria cutánea ya descrita que presentaba nuestra paciente no se observa en este síndrome y se excluyó una sobreposición con un síndrome de Sturge Weber que es relativamente frecuente en esta entidad, debido a que nuestra paciente no se evidenció en los estudios realizados angiomatosis leptomeníngea ni coroidea además que el nevus flammeus que presentó no era de distribución trigeminal.

El compromiso sistémico en la facomatosis pigmentovascular se ha asociado en el $50 \%$ de los casos, el cual incluye principalmente alteraciones en el sistema nervioso central, defectos oculares como melanosis oculi o glaucoma, hamartomas y mamilaciones del iris, alopecia, agenesia renal, síndrome de Klippel Trenaunay y el de Sturge Weber (11). Los pacientes que no presentan compromiso sistémico siguen un curso benigno y no requieren tratamiento específico. En algunos casos por motivo estético, se puede utilizar láser como el de Luz pulsada para tratar los nevus flammeus y Q switched para los nevus pigmentados, con buenos resultados (12).

El compromiso neurológico en la facomatosis pigmentovascular se ha reportado con la presencia de malformaciones de la unión cráneo - cervical como la entidad de Arnold Chiari, calcificaciones intracerebrales, atrofia cerebral, aplasia o hipoplasia de los vasos sanguíneos intracraneales, siendo los del terrritorio de la circulación posterior o vertebrobasilar los vasos más frecuentemente afectados (5).

La epilepsia es el compromiso neurológico más frecuente de los casos publicados hasta la fecha, la misma ha sido reportada como aislada o en la mayoría en asociación con anormalidades vasculares intracraneales como en el síndrome de Sturge Weber. En los casos descritos no se ha especificado el tipo de crisis epiléptica presentada en esta patología $(5,13)$. A la fecha se desconoce cuál sería la fisiopatología por la cual se presenta epilepsia asociada a esta entidad, habiéndose planteado como hipótesis que al ser la facomatosis pigmentovascular un síndrome neurooculocutáneo y que estos tres órganos tienen como origen el ectodermo, la migración anormal de las células derivadas de la cresta neural originarían una regulación neural anormal además de un desarrollo vascular aberrante lo que podría ocasionar microdisplasias corticales como ocurriría con otros síndromes cutáneos que están asociados a epilepsia como el síndrome PHACE y el síndrome de Sturge Weber; siendo el desarrollo de este mecanismo y el fenómeno de "twin spots" también la posible explicación de la asociación común entre este último síndrome y la facomatosis pigmentovascular. $(5,9$ $-10)$.

En nuestro caso se presentó una hipoplasia de la arteria comunicante posterior derecha y además de un síndrome epiléptico. Precisamente la paciente fue derivada a nuestra unidad de epilepsia con el diagnóstico de epilepsia mioclónica juvenil farmacorresistente y luego de ampliar la anamnesis y realizar el estudio de video electroencefalograma se concluyó que se trataba de una epilepsia focal mioclónica con una zona epileptogénica frontal mesial; siendo descartado el diagnóstico inicial de referencia.

La exacerbación de las crisis epilépticas estuvo relacionada, entre otros factores, temporalmente al consumo de marihuana, una vez retirada la sustancia psicoactiva, y con optimización del 
tratamiento antiepiléptico se logró el control de las crisis epilépticas. El tetrahidrocannabinol, una de las sustancias piscoactivas de la marihuana, puede disparar crisis epilépticas $(14,15)$. En este caso la pseudofarmacorresistencia se debió a la pobre adherencia a los fármacos antiepilépticos, al uso de marihuana (probablemente una cepa rica en tetrahidrocanabinol) y a la supresión del sueño.

En conclusión, reportamos por primera vez en Perú un caso de facomatosis pigmentovascular con compromiso neurológico; se resalta la importancia de una evaluación multidisciplinaria y oportuna en este tipo de patologías para descartar la presencia de compromiso extracutáneo que va a determinar el pronóstico del paciente (4). Además, este caso nos permite ilustrar que una adecuada anamnesis apoyada por el video electroencefalograma permite una óptima caracterización de la epilepsia y la identificación de pseudofarmacorresistencia.

\section{Correspondencia:}

Dr. Miguel Angel Vences Mijahuanca

Hospital Nacional Edgardo Rebagliati Martins, Departamento de Neurología

Av. Rebagliati 490 Jesús María

Correo electrónico: vens1793@hotmail.com

ORCID ID: https://orcid.org/0000-0002-8538-6242

\section{REFERENCIAS BIBLIOGRÁFICAS}

1. Ota M, Kawamura T, Ito N. Phakomatosis pigmentovascularis. Jpn J Dermatol. 1947; 57: 1-3.

2. Hasegawa $Y, \quad$ Yasuhara M. Phakomatosispigmentovascularis type IV a. Arch Dermatol. 1985; 121:651-652.

3. Torrelo A, Zambrano A, Happle R. Cutis marmorata telangiectasica congenita and extensive Mongolian spots: type $\mathrm{V}$ phakomatosispigmentovascularis. $\mathrm{Br} \mathrm{J}$ Dermatol. 2003; 148:342-5.

4. Happle R. Phacomatosis pigmentovascularis revisited and reclassified. Arch Dermatol. 2005;141: 385-388.

5. Fernández-Guarino M, Boixeda $\mathrm{P}$, De-Las-Heras E, et al. Phakomatosis pigmentovascularis: Clinical findings in 15 patients and review of the literature. $\mathrm{J}$ Am Acad Dermatol. 2008;58(1):88-93.
6. Vidaurri-De-La-Cruz H, Ruiz-Maldonado R. Facomatosis pigmentovascular. Hallazgos clínicos en los pacientes tratados en el Instituto Nacional de Pediatría 1971-2000. Tesis de Grado. Ciudad de México: Universidad Nacional Autónoma de México; 2012.

7. Cordisco MR, Campo A, Castro C, et al. Phakomatosis pigmentovascularis: report of 25 cases. Ped Derm. 2001; 18(4):70.

8. Happle R. Mosaicism in human skin, understanding the patterns and mechanisms. Arch Dermatol. 1993;129(11): 1460-70.

9. Happle R. New aspects of cutaneous mosaicism. J Dermatol. 2002; 29(11):681-92.

10. Thomas A, Zeng Z, Rivière JB, et al. Mosaic activating mutations in GNA11 and GNAQ are associated with phakomatosis pigmentovascularis and extensive dermal melanocytosis. J Invest Dermatol. 2016; 136(4):770-8.

11. Cadavid M, Zapata F, Velásquez N. Facomatosis pigmentovascularis asociada a síndrome de KlippelTrenaunay y a alopecia triangular: Reporte de caso. Rev CES Med. 2012; 26(2): 229-236.

12. Kono T, Erçöçen AR, Chan HH, et al. Treament of Phacomatosis pigmentovascularis: A combined multiple laser approach. Dermatol Surg. 2003; 29:642-6.

13. Nanda A, Al-Abdulrazzaq HK, Habeeb YK, et al. Phacomatosis pigmentovascularis: Report of four new cases. Indian J Dermatol Venereol Leprol. 2016; 82(3):298-303.

14. Yang H, de Jong JW, Tak Y, Peck J, Bateup HS, Lammel S. Nuccleus accumbens subnuclei regulate motivated behavior via direct inhibition and disinhibition of VTA dopamine subpopulations. Neuron. 2018; 97: 434-449.

15. Stockings E, Zagic D, Campbell G, et al. Evidence for cannabis and cannabinoids for epilepsy: a systematic review of controlled and observational evidence. J Neurol Neurosurg Psychiatry. 2018; 89: 741-53.

Recibido: 31/03/2020

Aceptado: 02/06/2020 